\title{
Adsorption of $\mathrm{C}_{2} \mathrm{H}_{2}-\mathrm{C}_{2} \mathrm{O}_{3}$ on $\mathrm{Si}(\mathbf{0 0 1})$
}

\author{
R. Miotto*, A. C. Ferraz ${ }^{\dagger}$, and G. P. Srivastava ${ }^{\dagger \dagger}$ \\ * Instituto de Física, Universidade de Brasília, Caixa Postal 04455, CEP 70919-970, Brasília, DF, Brazil \\ ${ }^{\dagger}$ Instituto de Física, Universidade de São Paulo, Caixa Postal 66318, CEP 05315-970, São Paulo, SP, Brazil \\ ${ }^{\dagger \dagger}$ School of Physics, University of Exeter, Stocker Road, Exeter, EX4 4QL, UK
}

Received on 31 March, 2003

\begin{abstract}
The adsorption of maleic anhydride on the $\mathrm{Si}(001)$ surface was investigated using the first-principles pseudopotential formalism. Our results indicate that $\mathrm{C}_{2} \mathrm{H}_{2}-\mathrm{C}_{2} \mathrm{O}_{3}$ adsorbs through a [2+2] cycloaddition of the $\mathrm{C}-\mathrm{C}$ bond to the dangling bonds of $\mathrm{Si}-\mathrm{Si}$ dimers, as observed for other small hydrocarbons. According to our calculations, the adsorption of a second maleic anhydride molecule will preferentially occur on first-neighbours $\mathrm{Si}-\mathrm{Si}$ dimer sites in either the dimer chain our dimer row, i.e. considering a $(4 \times 2)$ reconstruction, $\mathrm{C}_{2} \mathrm{H}_{2}-\mathrm{C}_{2} \mathrm{O}_{3}$ can either form a linear chain or a zig-zag chain, depending on the growth conditions. Although both structures are found to be stable from the energetic point of view, only our STM theoretical images for the linear chain are comparable to available experimental data.
\end{abstract}

\section{Introduction}

The increasing relevance of organic films in a great number of technological applications has motivated several investigations of the interaction of organic molecules with semiconductor substrates, specially silicon. It has been recently suggested [1] that the functionality associated with maleic anhydride can be used as controlled bonding sites for linking other organic substituents. Recent experimental investigations by Bitzer and co-workers [1, 2, 3] indicate that, depending on its coverage, $\mathrm{C}_{2} \mathrm{H}_{2}=\mathrm{C}_{2} \mathrm{O}_{3}$ can adsorb either on inter-dimer sites (or $[1+2+1]$ cycloaddition as in Fig. 1(b)) or through [2+2] cycloaddition (Fig. 1(a)). Our aim in this work is to investigate the adsorption process of maleic anhydride on the silicon surface. For doing so, we employ the state or art pseudopotential method, within a generalized gradient approximation to the density functional theory to investigate different adsorption sites for the maleic anhydride on the silicon surface.

The surface was modelled in a super-cell geometry, with an atomic slab of six Si layers and a vacuum region equivalent to eight atomic layers. On the top side of the slab we placed the $\mathrm{C}_{2} \mathrm{H}_{2}=\mathrm{C}_{2} \mathrm{O}_{3}$ molecule in different configurations, and the back surface was passivated by $\mathrm{H}$ atoms arranged in a dihydride structure. The pseudopotentials for $\mathrm{Si}, \mathrm{C}, \mathrm{O}$ and $\mathrm{H}$ were derived by using the scheme of Troullier and Martins [4] and the electron-electron exchangecorrelation interactions were considered by using a generalized gradient approximation (GGA) [5] of the density functional theory. The single-particle orbitals were expressed in a plane-wave basis up to the kinetic energy of $35 \mathrm{Ry}$. For the Brillouin-zone summation, four special $\mathbf{k}$ points were used. The atoms were assumed to be in their fully relaxed positions when the forces acting on the ions were smaller than

\section{$0.005 \mathrm{eV} / \AA$.}

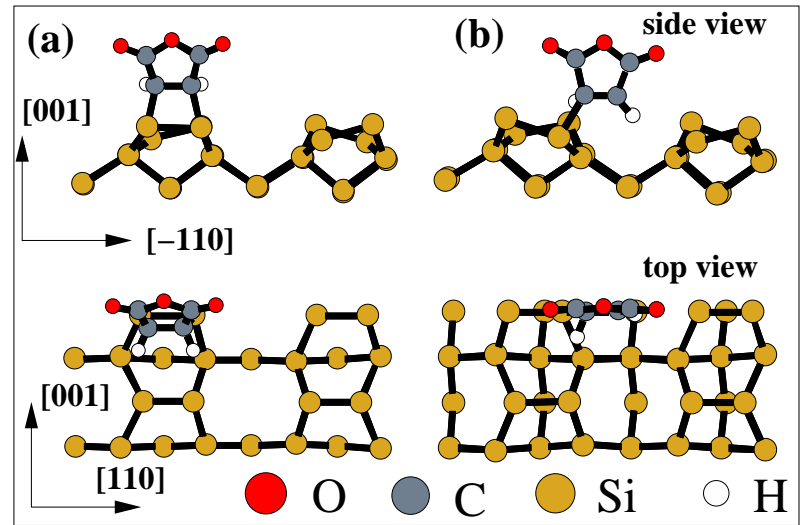

Figure 1. Schematic view of possible adsorption models for $\mathrm{C}_{2} \mathrm{H}_{2}=\mathrm{C}_{2} \mathrm{O}_{3}$ on $\mathrm{Si}(001)-(2 \times 4)$ : a) [2+2] cycloaddition, b) interdimer.

\section{Results}

For bulk silicon our first-principles calculations produced $5.50 \AA$ for the equilibrium lattice constant $\left(a_{o}\right), 0.86 \mathrm{GPa}$ for the bulk modulus $(B M)$, and $4.39 \mathrm{eV}$ for the cohesive energy, all in good agreement with the the experimental values presented in Ref. [6]. The calculated theoretical lattice constant obtained for the bulk silicon is used in surface calculations. Our calculations support the tilted $\mathrm{Si}-\mathrm{Si}$ dimer model that characterizes the clean $\mathrm{Si}(001)-(2 \times 1)$ surface: the $\mathrm{Si}-\mathrm{Si}$ dimer is found to have a bond length of $2.30 \AA$ and a vertical buckling of $0.73 \AA$, indicating a tilt angle of $17.8^{\circ}$. Our calculated bond lengths for the maleic anhydride 
molecule are $\mathrm{C}-\mathrm{C}=1.37 \AA$ for the double bond and $1.52 \AA$ for the single bond, $\mathrm{C}-\mathrm{O}=1.37 \AA$ for the double coordinated oxygen and $1.19 \AA$ for the single coordinated one, and $\mathrm{C}-$ $\mathrm{H}=1.10 \AA$, in good agreement with available experimental data [6].

Our first-principles calculations indicate that, for small coverages ( 1 molecule per $\mathrm{Si}-\mathrm{Si}$ dimer, or 0.125 monolayers (ML)) $\mathrm{C}_{2} \mathrm{H}_{2}=\mathrm{C}_{2} \mathrm{O}_{3}$ adsorbs via a [2+2] cycloaddition reaction, via the $\mathrm{C}=\mathrm{C}$ functionality (Fig. 1(b)), with an adsorption energy of $42 \mathrm{kcal} / \mathrm{mol}$. This is in agreement with the experimental findings of Bitzer and co-workers [2] but is slightly larger than the $31 \mathrm{kcal} / \mathrm{mol}$ calculated by Hofer et al [7] in a very recent calculation. This difference is probably related to the small size of the cell used in their work. In contrast to the experimental suggestions by Bitzer and co-workers [3], the adsorption on an inter-dimer site (Fig. 1(a)) is found to be energetically unfavourable by almost $30 \mathrm{kcal} / \mathrm{mol}$ when compared to the free molecule and the bare $\mathrm{Si}(001)$ surface. This is also against the indirect calculations by Hofer et al [7] based on elasticity theory. We understand that the main problem in Hofer's calculation is related to the strain energy obtained using a small cell, as this small cell does not take in consideration dimer-dimer interactions, for example.

As the indication of a inter-dimer adsorption was based on STM images [3], we show in Fig. 2 theoretical STM images for both the [2+2] cycloaddition and the inter dimer models. It is worthy pointing out that the present theoretical images are much richer than the experimental ones. If only the brighter spots are considered, our data suggest that it would be very difficult to differentiate between the considered models, as in both cases the adsorbates appear to be dislocated. Therefore we understand that our total energy calculations are consistent with the available experimental STM images.

\section{Conclusion}

Our total energy calculations suggest that, for low coverages, maleic anhydride adsorbs preferentially via a [2+2] cycloaddition directly over a Si-Si dimer and not in inter dimer sites, as recently suggested by Bitzer and co-workers [3, 7]. Calculated STM images for the both adsorption models are presented and compared to recent experimental observations with a view to support the proposed adsorption model.

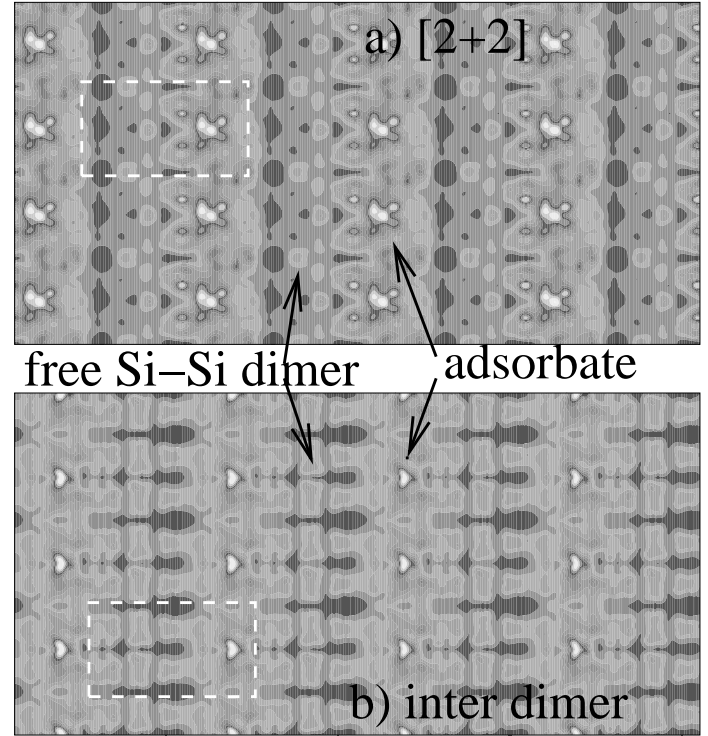

Figure 2. Calculated STM images for occupied states (bias voltage of $2.7 \mathrm{~V}$ ) for the adsorption of $\mathrm{C}_{2} \mathrm{H}_{2}=\mathrm{C}_{2} \mathrm{O}_{3}$ on $\mathrm{Si}(001)-(2 \times 4)$ considering a) $[2+2]$ cycloaddition $(0.125 \mathrm{ML})$ and b) inter-dimer. The dashed rectangle indicates a $(4 \times 2)$ reconstruction.

\section{Acknowledgments}

R. Miotto acknowledges financial support from FINATEC, DPP-UnB and CNPq.

\section{References}

[1] A. Lopez, T. Bitzer, T. Heller, and N. V. Richardson, Surf. Science 477, 219 (2001).

[2] T. Bitzer, T. Dittrich, T. Rada, and N. V. Richardson, Chem. Phys. Lett. 331, 433 (2000).

[3] T. Bitzer, T. Rada, and N. V. Richardson, J. Phys. Chem. B 105, 4535 (2001).

[4] N. Troullier and J. L. Martins, Phys. Rev. B 43, 1993 (1991).

[5] J. P. Perdew, K. Burke, and M. Ernzerhof, Phys. Rev. Lett. 77, 3865 (1996).

[6] D. R. Lide, Handbook of Chemistry and Physics (Chemical Rubber Company, Roca Raton, 1995).

[7] W. A. Hofer, A. J. Fisher, T. Bitzer, T. Rada, and N. V. Richardson, Chem. Phys. Lett. 355, 347 (2002). 